\title{
Development of Robust Fault Signatures for Battery and Starter Failure Prognosis
}

\author{
Xinyu $\mathrm{Du}^{1}$ and Yilu Zhang ${ }^{2}$ \\ ${ }^{1}$ General Motors Research \& Development, Warren, MI, 48363, USA \\ xinyu.du@gm.com \\ ${ }^{2}$ General Motors Research \& Development, Warren, MI, 48363, USA \\ yilu.zhang@gm.com
}

\begin{abstract}
Battery and starter are crucial vehicle components, whose failures may cause customers to be stranded. To enhance customer satisfaction and improve dealership serviceability, the failure prognosis and fault isolation for battery or starter are very important. In order to develop a robust diagnostic and prognostic solution, in this work, the feature extraction algorithms are developed to extract two fault signatures, namely battery charging resistance equivalent and battery cranking resistance ratio. The algorithms are based on the equivalent circuit model for the battery and starter system, the battery empirical model, and the field knowledge about the driver's behavior and battery management system. The proposed solution is a passive approach, and does not require any additional sensors for GM vehicles, or expensive computing hardware. Therefore, it is suitable for both onboard and off-board implementation. The solution has been validated with large fleet of vehicles under different scenarios, and implemented for selected GM vehicles through the OnStar ${ }^{\mathrm{TM}}$ Proactive Alerts service.
\end{abstract}

\section{INTRODUCTION}

An internal combustion engine has to be rotated by external force before it's able to run with its own power. Such a process is called cranking, which is performed by the vehicle starting system. The vehicle starting system normally consists of a battery, a starter, multiple electronic control units (ECUs) and other components. Here, the modern starter is a permanent magnet direct current motor with a relay-like solenoid mounted on it. The battery, commonly a $12 \mathrm{~V}$ leadacid battery, drives the starter motor to crank the engine. With the fuel injected and ignited, the engine is able to run sustainably. Malfunction of a component in the starting

Xinyu Du et al. This is an open-access article distributed under the terms of the Creative Commons Attribution 3.0 United States License, which permits unrestricted use, distribution, and reproduction in any medium, provided the original author and source are credited. system may cause vehicle no-start, and consequently lead to walk-home scenarios for customers.

The wear-out of $12 \mathrm{~V}$ battery is one of the leading reasons of customer dissatisfaction in the automotive industry. To enhance customer ownership experience, GM developed and launched OnStar ${ }^{\mathrm{TM}}$ Proactive Alerts service in 2015 on selected GM vehicles. This service predicts the incoming failures of several critical vehicle components, including $12 \mathrm{~V}$ battery and starter, before the vehicle performance is affected.

Failure prognosis has positive impacts to original equipment manufacturer (OEM) warranty cost as well. Modern vehicle design is very complex. It is increasingly challenging to identify root cause of vehicle failures. As a result, many healthy components are replaced at repair shops unnecessarily, which causes a high rate of incidence of no trouble found (NTF). A pre-requisite of failure prognosis is the accurate identification of root cause of potential failures, which will reduce NTF.

In the area of battery and starter prognosis, many research papers and patents have been published, mainly in four categories, battery SOH estimation, battery state of charge (SOC) estimation, state of function (SOF) estimation for starting system, and remaining useful life (RUL) estimation (Cuma \& Koroglu, 2015) (Farmann, Waag, Marongui, \& Sauer, 2015) (Berecibar, et al., 2016). A diagnostic and prognostic scheme always includes two critical steps, feature extraction and reasoning. For the first step, the useful sensor data, which are correlated to the system failures, are identified and selected from all available signals. The features, i.e. the fault signatures, are developed or extracted from the selected sensor signals. The battery internal resistance or impedance is a common feature for battery $\mathrm{SOH}$ estimation. It's generally estimated using an equivalent circuit model (Pilatowicz, Marongiu, Drillkens, Sinhuber, \& Sauer, 2015) or an electrochemical model (Zhang, Du, \& Salman, Battery state estimation with a self-evolving 
electrochemical ageing model, 2017). Other features to predict failures are also employed in different literature, e.g. the back EMF (electromotive force) voltage for SOC estimation (Coleman, Lee, Zhu, \& Hurley, 2007), “coup de fouet" for SOC estimation, OCV-SOC mapping table for SOC estimation (Zhang, Du, \& Salman, Battery state estimation with a self-evolving electrochemical ageing model, 2017), and minimum cranking voltage for SOF estimation (Zhang, Grube, Shin, Salman, \& Conell, 2011). The reasoning logic is to map feature data to the exact failure mode, provide trending analysis and final prognostic decisions. The logic can be a heuristic decision tree, a datadriven black box or a physical model. The reasoning approaches found in the literature include sample entropy and sparse Bayesian predictive modeling approach (Hu, Jiang, Cao, \& Egardt, 2016) for RUL estimation, Lebesguesampling-based battery state of health $(\mathrm{SOH})$ diagnosis (Yan, Zhang, Wang, Dou, \& Wang, 2016), Kalman filter for SOC estimation (Wang, Fang, Wang, \& Liu, 2016), prognosis of gear failures in DC starter motors using hidden Markov Models (Zaidi, Aviyente, Salman, Shin, \& Strangas, 2011), Hळ Observer for SOC estimation (Zhang, Liu, Fang, \& Wang, 2012), and artificial neural network for starter motor diagnosis (Bayir \& Bay, 2004). In GM R\&D, the vehicle health management group has done substantial work in this area (Zhang, Grube, Shin, Salman, \& Conell, 2011) (Shin \& Sabahi-Kaviani, Diagnosis and Prognosis of Cranking Systems: A Parameter Based Approach, 2010) (Shin \& Salman, Evidence theory based automotive battery health monitoring, 2010). Various new fault signatures, including delta voltage, cranking energy, cranking time are proposed, and the least-square-based filtering algorithms are developed to extract these features. The Dempster-Shafer evidence theory and the parity approach are proposed to detect and isolate failures for the vehicle starting system.

However, there is still no accurate and reliable production solution to predict battery or starter failures in the automotive industry before OnStar ${ }^{\mathrm{TM}}$ Proactive Alerts. There are various reasons. First, certain existing approaches require excess excitations (Cugnet, et al., 2010), which are available in the laboratory environment or electrical vehicles, but not for conventional vehicles with an internal combustion engine. During driving, the conventional vehicles charge the battery most of time, which means there is not enough data to evaluate the battery discharging performance. Cranking is a good source but it only takes about 1 second. The sensor to rapidly capture all the data during cranking is expensive. Second, the vehicle lead-acid battery is highly dynamic. The phenomenon of surface charge and acid stratification may impact fault signatures, e.g. battery resistance or battery open circuit voltage, which may lead to false alerts. The performance of a lead acid battery is impacted by the driving behavior and environment as well. A battery, not used for a couple of months, may exhibit similar performance as a battery at the end of the life. However, such a battery can perform normally after several ignition cycles.

To develop a robust prognostic algorithm and address all these challenges, the key is the big data from the field. The data can help us to better understand the system, calibrate and refine algorithms accordingly. Recently, the telematics technology grows rapidly, and the vehicle data from a large fleet of vehicles can be collected and processed easily with low cost. In GM R\&D, this direction has been explored since 2010 (Zhang, Du, \& Salman, Peer-to-peer collaborative vehicle health management - the concept and an initial study, 2012) (Zhang \& Du, Automatic field data analyzer for closed-loop vehicle design, 2014). A collaborative vehicle health management system is proposed to adaptively calibrate the estimation algorithm at run time based on the V2X technology after the vehicle deployment. The preliminary experiment results using several test vehicles shows the effectiveness of this scheme. With the OnStar ${ }^{\mathrm{TM}}$ gen 10 module equipped in the vehicle, 4G LTE technology can be employed to quickly transmit vehicle data to back office. All these changes make a robust starting system prognosis become possible.

As the first step, in this work, several fault signatures are proposed and the robust algorithms to extract these features are developed. The proposed solution only utilizes existing vehicle signals, and doesn't require any additional sensors or onboard changes. The proposed approaches are validated using more than 600,000 vehicle data with about 1 year driving. The remainder of this paper is structured as follows. The development of the fault signature cranking resistance ratio is presented in Section 2. The fault signature charging resistance equivalent is described in Section 3. The conclusion and future directions are discussed at the end.

\section{Cranking Resistance Ratio}

When the internal resistance of a battery is increased, the battery can't provide enough power to drive the starter motor. On the other hand, when the starter resistance is increased, the current through the starter motor is decreased, and the torque that the starter can provide is reduced as well. Both scenarios may lead to a no-start event. Therefore, the battery resistance and the starter resistance during cranking is an important indicator to predict the battery or starter failures.

\subsection{Modeling and Analysis}

For a typical cranking process, the battery voltage starts at around 12.5 volts, and decreases rapidly once the ignition starts. The voltage reaches the minimum value around $7.5 \mathrm{~V}$ at 0.1 second. At the same time, the discharging current reaches its maximum value. At this particular timestamp, the derivative of the current is close to 0 , and the starter doesn't spin. Therefore, the voltage induced by back EMF can be ignored. The battery and starter circuit can be simplified as a pure resistance network, shown in Fig. 1. 


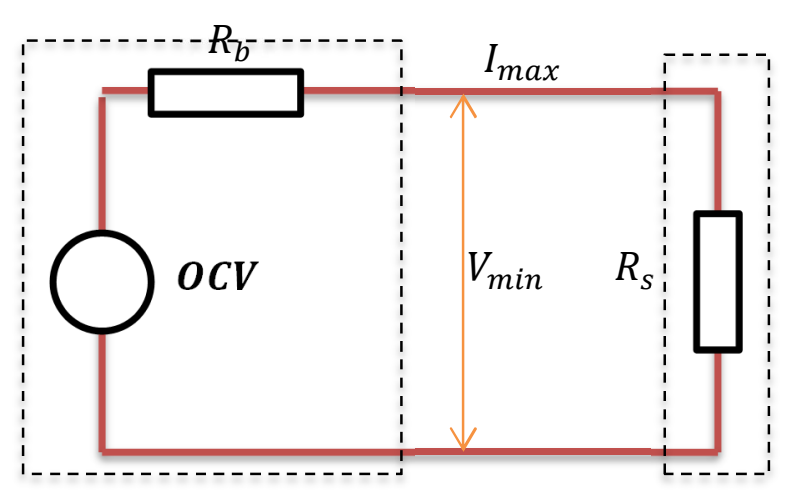

Figure 1. The equivalent circuit model for the battery and the starter when the cranking voltage is at its minimum value.

Based on the equivalent circuit model, the battery current can be calculated as follows,

$$
I_{\max }=\frac{O C V-V_{\min }}{R_{b}}=\frac{V_{\min }}{R_{S}}
$$

where $R_{b}$ is battery resistance, $R_{s}$ is starter resistance, $V_{\text {min }}$ is the minimum cranking voltage, and $I_{\max }$ is the battery current at the time stamp of minimum cranking voltage. Generally $I_{\max }$ is not available from the vehicle, therefore, the battery resistance and the starter resistance can't be calculated, respectively. From the right two terms of the equation (1), the minimum cranking voltage can be calculated as below,

$$
V_{\min }=\frac{O C V \times R_{S}}{R_{b}+R_{S}}=\frac{O C V}{R_{b} / R_{S}+1}
$$

The ratio between the battery resistance and the starter resistance is defined as cranking resistance ratio $r$, that is,

$$
R_{b} /_{R_{S}} \triangleq r=\frac{O C V}{V_{\text {min }}}-1
$$

From the basic physical characteristics of batteries and starters, both the battery resistance and the starter resistance are impacted by temperature. When the temperature decreases, the battery internal resistance increases, and the starter resistance decreases. Therefore, we can normalize the cranking resistance ratio to the temperature using the following empirical equation,

$$
r_{0}=r \times \frac{1+\beta\left(T_{s}-25\right)}{1-\alpha\left(T_{b}-25\right)}
$$

where $T_{s}$ is the estimated starter temperature, and $T_{b}$ is the estimated battery temperature. The battery temperature is available in the vehicle as a PID. The starter temperature is not available. But it can be estimated using a Lumped thermal mass model as follows,

$$
T_{s}=\left\{\begin{array}{c}
T_{b}+a e^{b \cdot t_{o f f}, \text { if } t_{o f f} \leq T h 1} \\
T_{b}, \text { if } t_{o f f}>T h 1
\end{array}\right.
$$

where $\alpha$ and $\beta$ are temperature coefficients for the battery and the starter, respectively. $a$ and $b$ are fitting coefficients. $T h 1$ is a predefined threshold. $t_{o f f}$ is the engine off time. This empirical model is developed based on the field knowledge. The starter is attached to the engine, and made of metal. Therefore, its specific heat capacity is low. The majority of materials of a lead-acid battery are sulfuric acid, whose specific heat capacity is high. When the engine-off time is long enough (greater than Th1), the starter temperature is closed to the battery temperature, which is closed to the ambient temperature. If the engine-off time is short, the starter temperature is higher than the battery temperature since the engine temperature is higher than the battery temperature.

In addition to the equation (5), the empirical model can also be, but not limited to, the following two models,

Linear model: $\boldsymbol{f}\left(\boldsymbol{T}_{\boldsymbol{b}}, \boldsymbol{t}_{\boldsymbol{o f f}}\right)=a \boldsymbol{T}_{\boldsymbol{b}}+b \boldsymbol{t}_{\boldsymbol{o f f}}+c$

Lumped thermal mass model with linear approximation:

$$
\boldsymbol{f}\left(\boldsymbol{T}_{\boldsymbol{b}}, \boldsymbol{t}_{\text {off }}\right)=\boldsymbol{T}_{\boldsymbol{b}}+a \boldsymbol{t}_{\text {off }}+b
$$

where $a, b$ and $c$ are fitting coefficients.

\subsection{Algorithms}

Based on the above analysis, an algorithm is developed to estimate the normalized cranking resistance ratio. The algorithm is triggered every ignition cycle. After the vehicle cranks, the battery start-up SOC, the battery estimated temperature, the engine-off time, and the minimum cranking voltage are collected. From our test results, the data collection should not happen right after the cranking since it takes some time for ECUs to update their PID values. For the data used in this work, the data are collected after five minutes of cranking.

Once the data are collected, a filtering algorithm is executed to remove outliers. For example, one outlier at $11 \mathrm{~V}$ is found among continuous more than 300 sessions from a vehicle driving history. All other data points are normal between $8 \mathrm{~V}$ and $9 \mathrm{~V}$. Since both the battery and the starter are in a healthy state for the whole period based on all vehicle signals and warranty transaction records, the data point at $11 \mathrm{~V}$ is obviously an outlier. The outlier may be due to the sensing circuit error, software bugs or other reasons. The outlier has to be removed since it may trigger false positive. To remove the outlier, the current minimum cranking voltage needs to be compared to the previous values. If the current minimum cranking voltage value is too high or too low, which is physically impossible, or marginally high but apparently different from the neighboring points, the point can be identified as an outlier.

After removing all outliers, if the data are not enough (e.g. the number of the data is less than 3 ) to calculate the cranking resistance ratio, a predefined value, which represents this scenario, will be assigned to the ratio. If the data is sufficient, 
the start-up SOC needs to be checked if it's a default value. In various scenarios, e.g. low SOC, cable disconnect, extreme low voltage, the start-up SOC may be reset to a default value. In this case if the minimum cranking voltage is also very low the battery is likely to be a bad battery. Therefore, a predefined high value is assigned to the cranking resistance ratio to increase the algorithm coverage.

If all required signals are available and not default, the OCV in equation (3) should be calculated using the start-up SOC and the OCV-SOC mapping table. This is because the OCV value saved in vehicle is updated during ignition-off, which is not equal to the actual OCV during cranking.

With the OCV and the minimum cranking voltage, the cranking resistance ratio can be calculated using equation (3). The starter temperature during cranking can be estimated using the equation (5). The normalized cranking resistance ratio can be calculated using equation (4).

\subsection{Performance Evaluation and Comparison}

In order to evaluate the performance of the cranking resistance ratio algorithm, a metric, called range-standarddeviation-ratio $\boldsymbol{R} / \boldsymbol{\sigma}$, is employed. It's similar to the signalnoise-ratio. Here, the range $\boldsymbol{R}$ is defined as the distance between the mean of healthy data and the mean of faulty data. Higher $\boldsymbol{R}$ means easier to isolate the fault. $\boldsymbol{\sigma}$ is the standard deviation of the data corresponding to the healthy state. Smaller $\boldsymbol{\sigma}$ indicates the signal is not noisy, and unlikely causes a false positive. Therefore, the larger $\boldsymbol{R} / \boldsymbol{\sigma}$ is, the better the isolation performance with this feature is.

Table 1 compares the $\boldsymbol{R} / \boldsymbol{\sigma}$ metrics for the data from 121 MY12/13 Equinox/Terrain vehicles with a LFX engine using different normalization methods. The percentage in bracket for each method is the improvement comparing to the baseline method (the cranking resistance ratio for the standard deviation, and the minimum cranking voltage for the range-standard-deviation-ratio). We can't use the minimum cranking voltage as the baseline for the standard deviation comparison since the unit is different between the voltage and the ratio. One can easily observe that the proposed approach, normalized cranking resistance ratio using both estimated battery temperature and estimated starter temperature (equation 5) is the best among all methods, and is $113 \%$ better than the minimum cranking voltage, in terms of the rangestandard-deviation-ratio.

To further illustrate the performance of these methods, the data for the cranking resistance ratio, the normalized cranking resistance ratio using the estimated battery temperature only, and the normalized cranking resistance ratio using both the estimated battery temperature and the estimated starter temperature from one vehicle are plotted in Fig. 2. The temperature dependency can be clearly observed for the original cranking resistance ratio. The standard deviation for the proposed method is the smallest.
Table 1 Performance comparison between different approaches in terms of the cranking resistance ratio and the minimum cranking voltage.

\begin{tabular}{|l|l|l|}
\hline Signals & $\boldsymbol{\sigma}$ & $\boldsymbol{R} / \boldsymbol{\sigma}$ \\
\hline Min Cranking Voltage & & $9.52(0)$ \\
\hline Cranking Resistance Ratio & $0.0329(0)$ & $14.49(52 \%)$ \\
\hline Normalized Ratio with Battery Temp & $0.0281(15 \%)$ & $16.17(70 \%)$ \\
\hline Normalized Ratio with Equation 6 & $0.0213(35 \%)$ & $19.79(108 \%)$ \\
\hline Normalized Ratio with Equation 5 & $0.0214(35 \%)$ & $20.25(113 \%)$ \\
\hline Normalized Ratio with Equation 7 & $0.0214(35 \%)$ & $20.11(111 \%)$ \\
\hline
\end{tabular}

The normalized cranking resistance ratio with the estimated battery temperature and the estimated starter temperature minimizes the temperature dependency. An alternative consideration is to normalize the ratio with the coolant temperature since the coolant temperature is close to the engine temperature and starter temperature. However as shown in Fig. 3, the temperature dependency for the original ratio is similar to the dependency for the normalized ratio with the coolant temperature.

Figs. 4-8 show the time series data from four vehicles for the raw cranking resistance ratio, the ratio normalized by the battery temperature, and the ratio normalized by both battery temperature, and the ratio normalized by both estimated battery temperature and estimated starter temperature. All the cranking resistance ratio data are normalized to 0 to 1 since

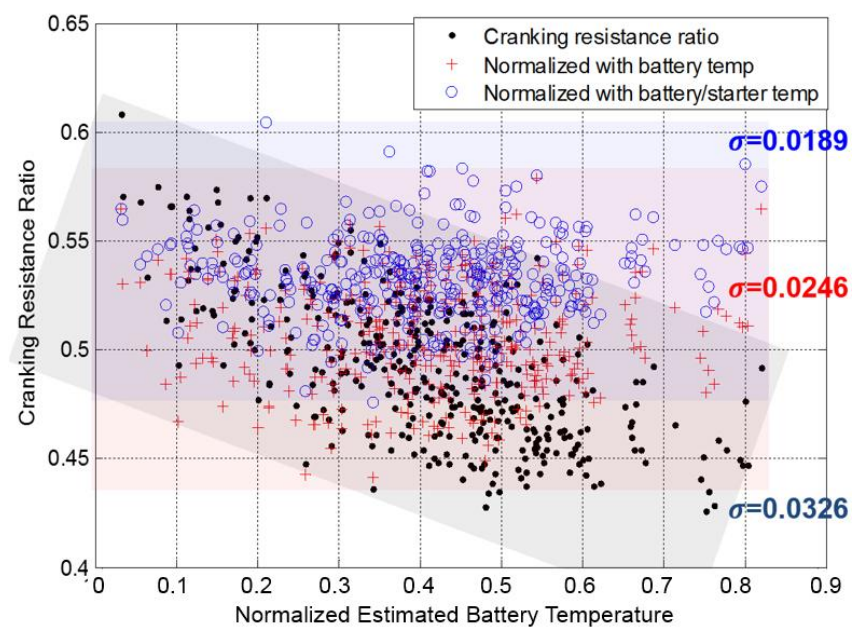

Figure 2. Comparison of cranking resistance ratio distribution for original cranking resistance ratio, normalized cranking resistance ratio using estimated battery temperature only, and normalized cranking resistance ratio using both estimated battery temperature and estimated starter temperature. The estimated battery temperature is normalized to 0 to 1 . 


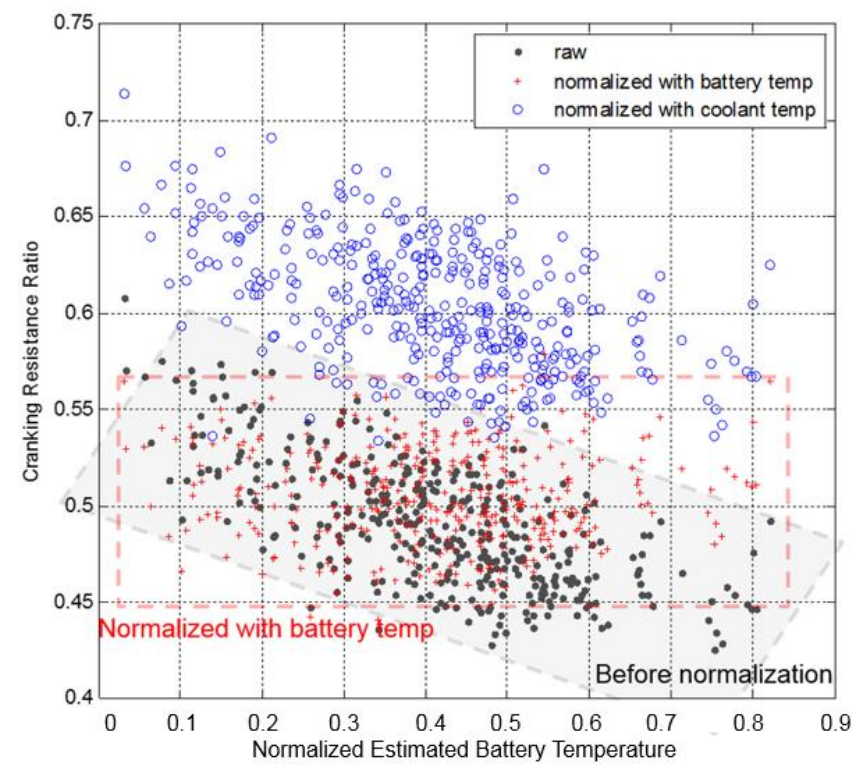

Figure 3. Comparison among the cranking resistance ratio, the cranking resistance ratio normalized with the estimated battery temperature, and the cranking resistance ratio normalized with the coolant temperature. The estimated battery temperature is normalized to 0 to 1 .

the data are sensitive. The red vertical line indicates the repair date in each figure. One can observe that right before the repair, the ratio is very high and after the repair the ratio is normal. In addition, the standard deviation for the proposed approach in the healthy region is the smallest among all these approaches.

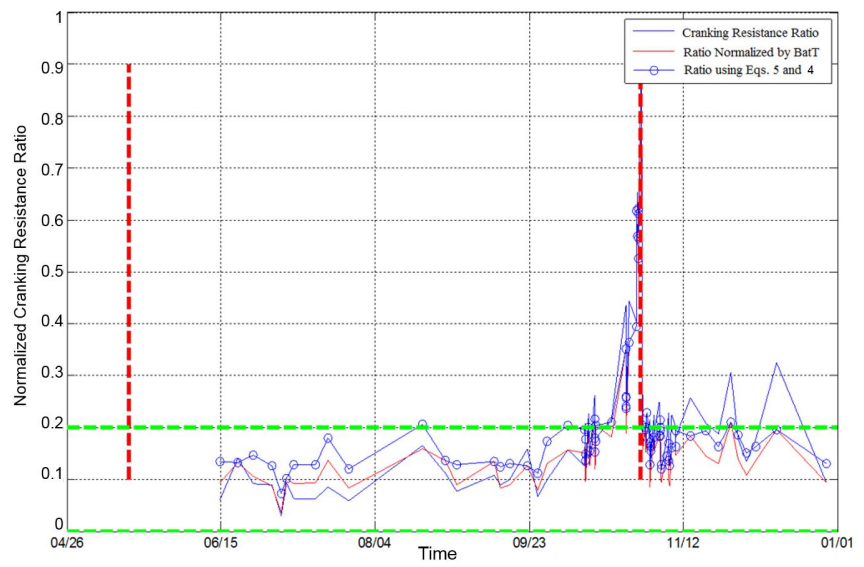

Figure 4. Comparison between different cranking resistance ratio calculation methods from the field data (Case 1). The blue line is the original ratio, red solid line is the ratio normalized by the battery temperature and the blue dot line is the ratio normalized by battery and starter temperature. The red vertical line indicates the repair date and the green line indicates the healthy region. The cranking resistance ratio is normalized to 0 to 1 . All apply to Figs. 5-8 as well.

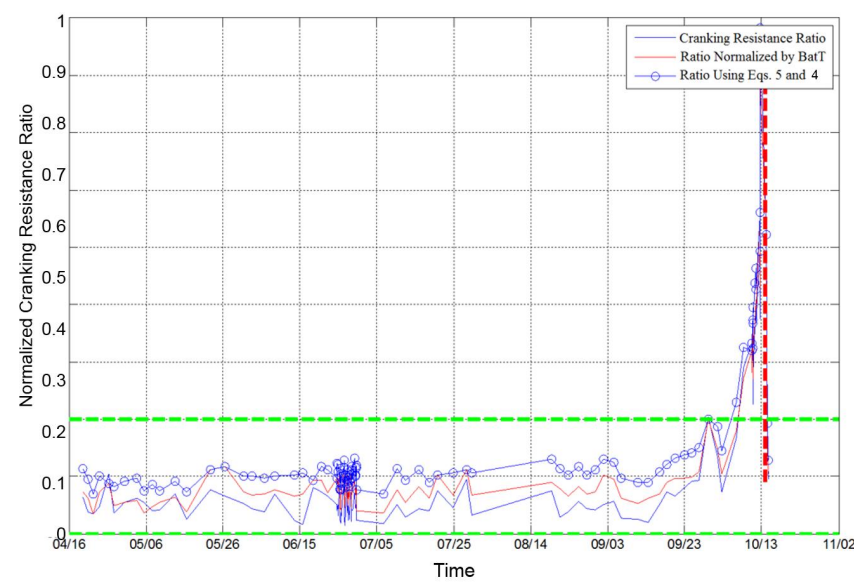

Figure 5. Comparison between different cranking resistance ratio calculation methods from the field data (Case 2).

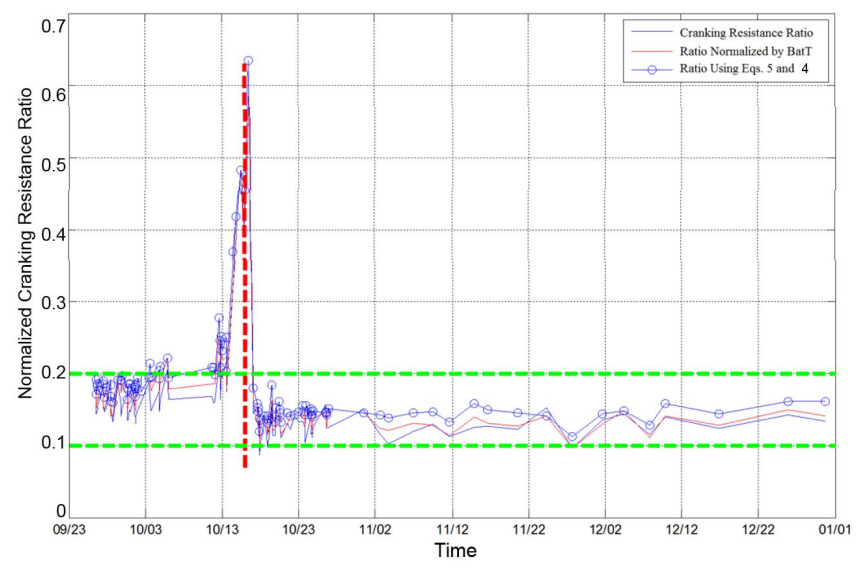

Figure 6. Comparison between different cranking resistance ratio calculation methods from the field data (Case 3).

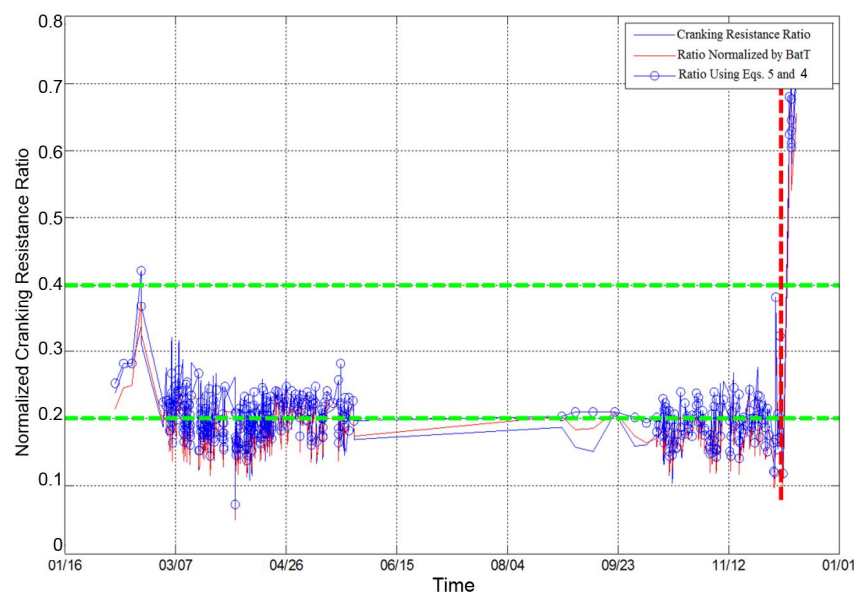

Figure 7. Comparison between different cranking resistance ratio calculation methods from the field data (Case 4). 


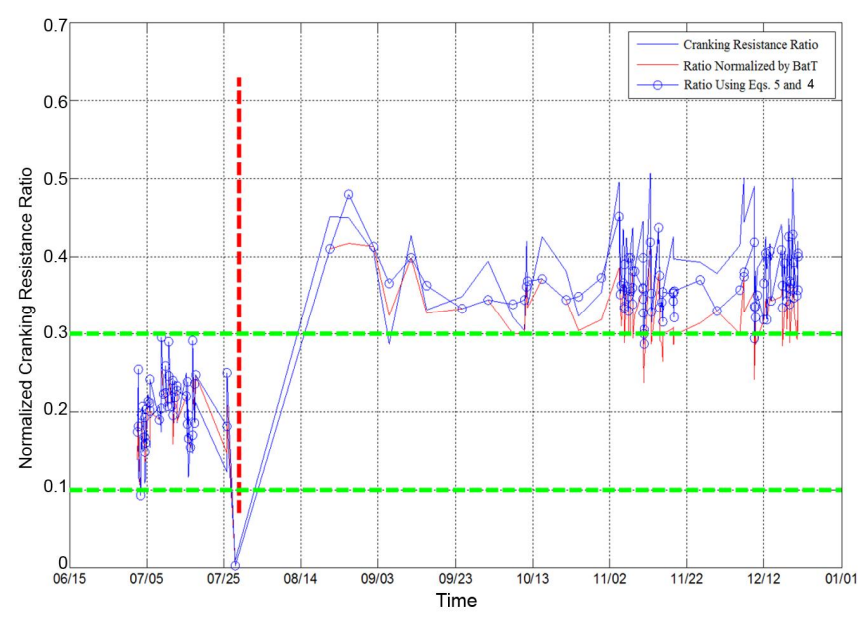

Figure 8. Comparison between different cranking resistance ratio calculation methods from the field data (Case 5).

\section{Charging Resistance Equivalent}

When a battery is at the end of its life, it becomes hard to be charged. Therefore, the charging resistance is a potential fault signature for a battery. Not like the cranking resistance ratio, which is affected by both battery and starter, this feature only indicates the battery failure. Please note that battery charging resistance depends on the charge acceptance, therefore, it's not a sufficient condition to determine battery failures. A battery with extremely high SOC also has low charge acceptance, although the battery may be good. Therefore, it's more meaningful to normalize the charging resistance by SOC. Please also note that the charging resistance is different from the cranking resistance that is discussed in Section 2, or the battery discharging resistance since the electrochemical reaction is different between charging and discharging.

\subsection{Modeling Battery Charging Resistance Using Shepherd Equation}

The battery charging process can be modeled with Shepherd equation (Shepherd, 1965) (Schiffer, et al., 2007),

$$
\begin{aligned}
& U(t)=U_{0}-g(1-\operatorname{SOC}(t))+\rho_{c} \frac{I(t)}{C_{N}}+ \\
& \rho_{c} \frac{I(t)}{C_{N}} M_{c} \frac{\operatorname{SOC}(t)}{C_{c}-\operatorname{SOC}(t)}, \quad \forall I(t)>0
\end{aligned}
$$

where $I(t)$ is the battery current, $\rho_{c}$ is the charging resistance equivalent $(\Omega \cdot A h)$, also called aggregated internal resistance in some references (Schiffer, et al., 2007), $C_{N}$ is the rated battery capacity, $g$ is the electrolyte proportionality constant, $M_{c}$ is the charge-transfer overvoltage coefficient (nominal value, 0.88$), C_{c}$ is normalized capacity ( 1 is for a new battery), $U(t)$ is the battery terminal voltage, and $U_{0}$ is the open-circuit voltage for the fully charged battery. Since $U_{0}-$ $g(1-S O C(t))$ is the battery OCV at the current SOC, Shepherd equation can be rewritten in the estimation form as follows,

$$
\rho_{c}=\frac{U(t)-O C V}{I(t)} \frac{C_{N}}{\left(1+M_{C} \frac{S O C(t)}{C_{C}-S O C(t)}\right)}
$$

The charging resistance equivalent $\rho_{c}$ can then be calculated from Equation (9). In order to better understand this equation, we replace $U(t)-O C V$ by $I(t) \times R+U_{D}$, where $R$ is the battery internal charging resistance, and $U_{D}$ is the voltage difference between the $O C V$ under discharging and the $O C V$ under charging (normally about $0.6 \mathrm{~V}$ ). The equation (9) becomes,

$$
\rho_{c}=\frac{I(t) R+U_{D}}{I(t)} \frac{C_{N}}{\left(1+M_{C} \frac{S O C(t)}{C_{C^{-}}-S O C(t)}\right)}=\left(R+\frac{U_{D}}{I(t)}\right) \frac{C_{N}}{\left(1+M_{c} \frac{S O C(t)}{C_{C^{-}}-S O C(t)}\right)}
$$

To better investigate the behavior of $\rho_{c}$, we consider a few simulated examples. For a brand-new battery with full charge, the SOC is around $100 \%$. Take SOC $=100 \%$ into the equation (10), $1+M_{c} \frac{\operatorname{SOC}(t)}{C_{c}-\operatorname{SOC}(t)}$ becomes a very high value. If $I(t)$ is not a value close to $0, \rho_{c}$ will be very small. This is consistent with our assumption that the battery SOH is high.

When the SOC is low, there are two possibilities, the battery is with low SOC but high $\mathrm{SOH}$, or with low SOC and low $\mathrm{SOH}$. Assume $\mathrm{SOC}=0 \%, 1+M_{c} \frac{\operatorname{SOC}(t)}{C_{c}-\operatorname{SOC}(t)} \cong 1, \rho_{c}=$ $R C_{N}+\frac{U_{D}}{I(t)} C_{N}$. If the $\mathrm{SOH}$ is high, the charging current should be very high since this is a low SOC battery. Assume $C_{N}=$ $80, U_{D}=0.6, I(t)=10$ and $R=0.005 \Omega, \quad \rho_{c}$ is calculated as 5.2. On the other hand, if the $\mathrm{SOH}$ is low, the battery charging current should be low too. Assume $I(t)=2$ and $R=0.008 \Omega, \quad \rho_{c}$ is calculated as 24.6.

When the SOC is medium, assume the $\mathrm{SOC}$ is $0.6, \rho_{c} \cong$ $\left(R C_{N}+\frac{U_{D}}{I(t)} C_{N}\right) / 2.32$. When the battery $\mathrm{SOH}$ is high, assume $I(t)=10$ and $R=0.005 \Omega, \rho_{c}$ is 2.2 . When the

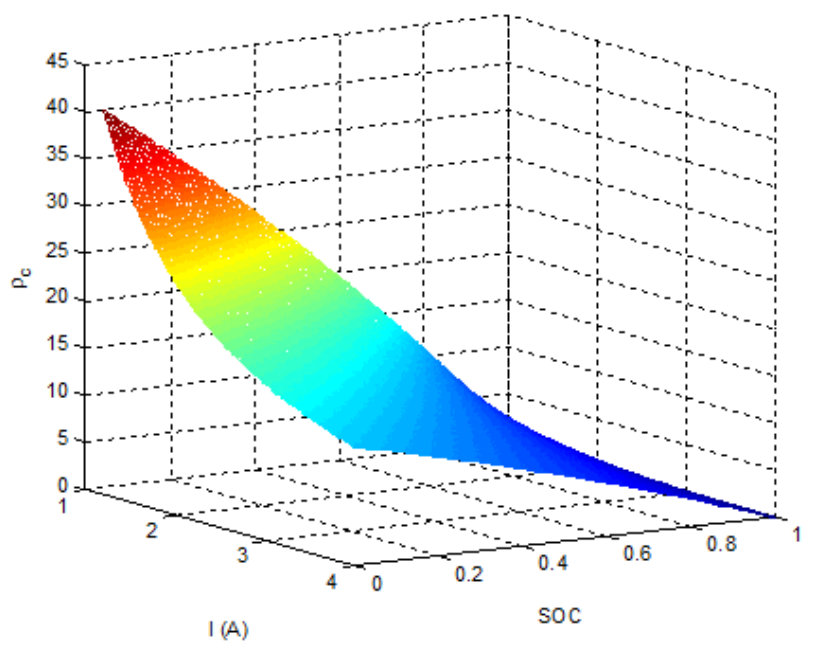

Figure 9. The illustrative relationship between the charging resistance equivalent, SOC and charging current when $M_{C}=$ $0.8, R=0.005 \Omega$ and $U_{D}=0.6 \mathrm{~V}$. 


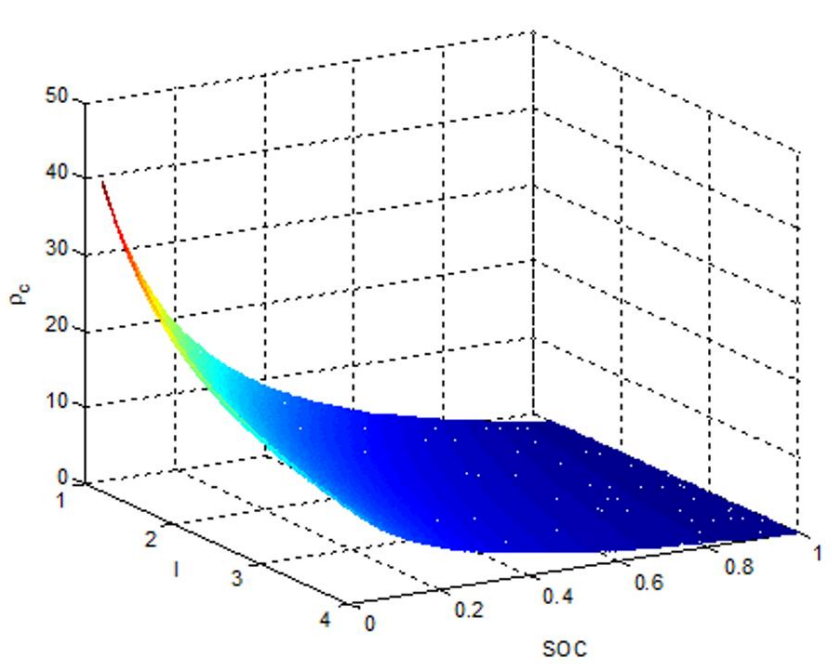

Figure 10. The illustrative relationship between the charging resistance equivalent, SOC and charging current when $M_{c}=$ $5, R=0.005 \Omega$ and $U_{D}=0.6 \mathrm{~V}$.

battery $\mathrm{SOH}$ is low, assume $I(t)=2$ and $R=0.008 \Omega$ $\rho_{c}$ is 10.6 .

In summary, when $\mathrm{SOC}=1$, no matter what the value of $M_{c}$ is, $\rho_{c}$ is almost 0 . When $\mathrm{SOC}=0$, no matter what the value of $M_{c}$ is, $\rho_{c}$ only depends on the current. When SOC and charging current are medium, a higher $M_{c}$ will lead to a higher $\rho_{c}$, which are shown in the Figs. 9 and 10. The low charging current is associated with the high charging resistance equivalent. And the high current is associated with the low charging resistance equivalent.

Please note the current $I(t)$ is in the denominator of the equation (10). When the current is very small and other signals, e.g. voltage and SOC, are not accurately measured or estimated, $\rho_{c}$ may be calculated abnormally high even though the battery is healthy. This will result in false positive decisions. To ensure the algorithm robustness, the low current data need to be filtered before the charging resistance equivalent is estimated.

\subsection{Algorithms}

With above analysis, an algorithm is developed to calculate the charging resistance equivalent described as below. The inputs include the battery SOC, the battery OCV, battery terminal voltages, and battery currents.

Multiple samples of voltages and currents may be out of order or some values may be missing due to data transmission. In this scenario, we need to align the voltage and current so that they are in the right order. First of all, all the points are stored in two vectors, EngName for the PID name (1 is used in the code for the voltage and 2 for the current) and EngValue for the PID value. If the length of two vectors is 0 , the empty vector is assigned to the aligned output vectors, BatV and BatI. Otherwise, an index vector $I_{1}$ is calculated to store the indexes for all points, whose EngName is equal to 1. Then for each point, whose EngName is equal to 1, if the next point is the current, this pair of voltage and current data is saved to the output vector BatV and BatI. The algorithm ends once all data points are processed.

After the alignment, if the data are not enough (e.g. the number of the data is less than 3), a default value, which represents this senario, is assigned to the charging resistance equivalent. Otherwise, the effective battery voltages and the effective battery currents are calculated by filtering out the points whose current value is less than the threshold $T h_{I c}$. The charging resistance estimate is more robust after filtering out the data with low current, which are shown in Figs. 11 and 12. If the number of data points after filtering are not enough, it indicates the battery current is very small. In this scenario, certain BCM reset conditions should be checked since the battery is likely not in a good condition, and may result in a reset.

In the scenario that the amount of effective battery voltage and current data is enough, equation (10) (the state estimation form) is applied to calculate the charging resistance equivalent.

\subsection{Performance}

The proposed algorithm has been validated using MY12/13 Equinox/Terrain vehicles with a LFX engine. Some of cases with the battery high resistance fault are shown in the Figs. 13,14 and 15 . The battery charging resistance is increased before the repair date, and becomes very small after the repair, which demonstrates this feature is effective to predict battery failures. Please note that the charging resistance estimate data are further normalized to 0 to 1 since the data are sensitive.

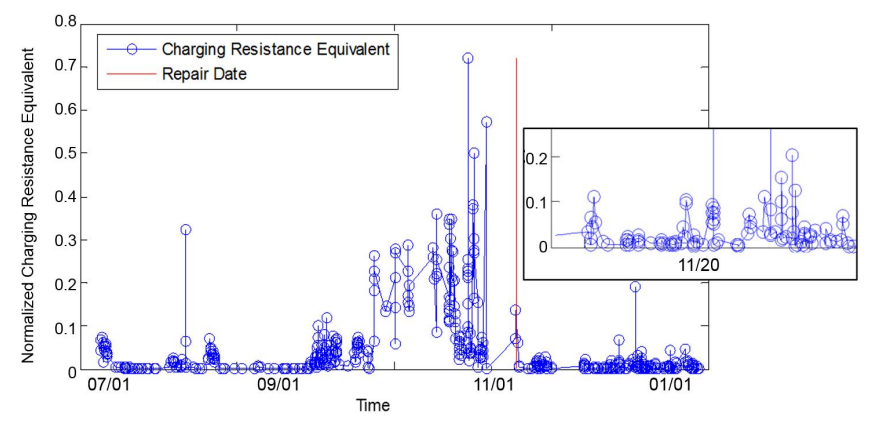

Figure 11 . The battery charging resistance equivalent when there is no current filtering, i.e. $T h_{I c}=0$. The small figure shows a detailed view after the replacement. 


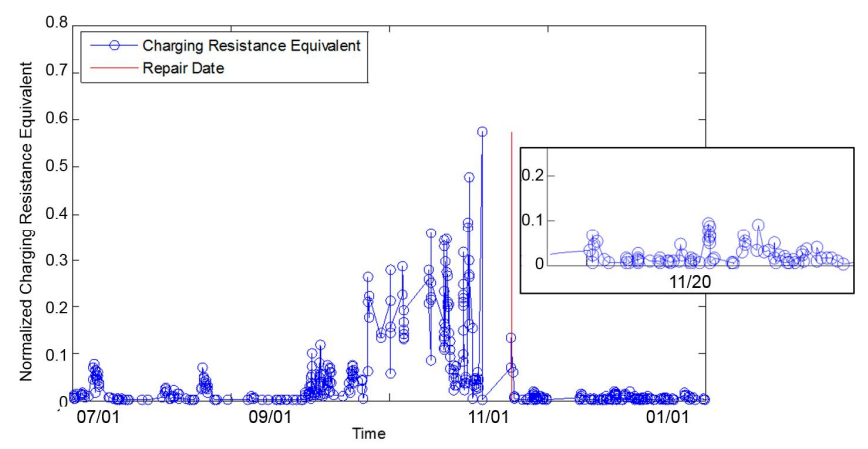

Figure 12 . The battery charging resistance equivalent when the cut off threshold is equal to $1 \mathrm{~A}$. The small figure shows a detailed view after the replacement

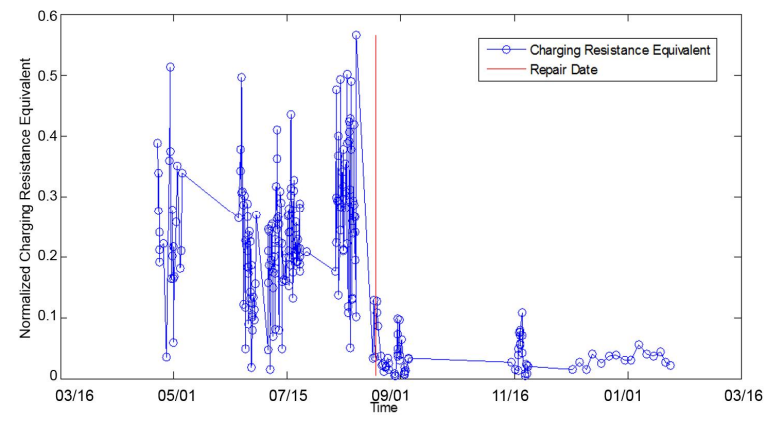

Figure 13. The trend of the battery charging resistance equivalent from one field case (Case 6).

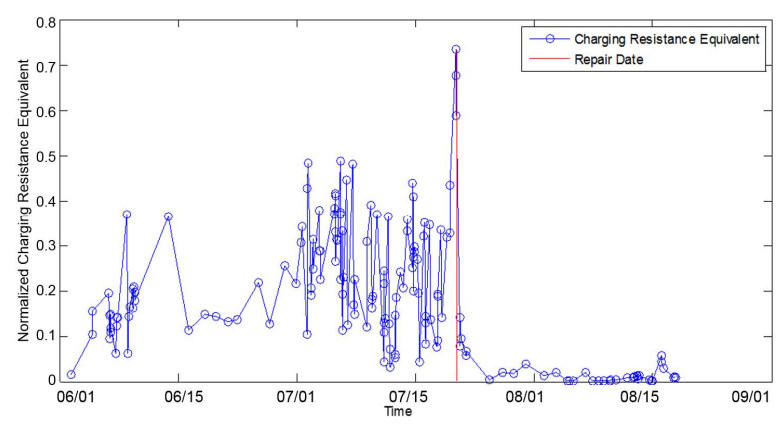

Figure 14. The trend of the battery charging resistance equivalent from one field case (Case 7).

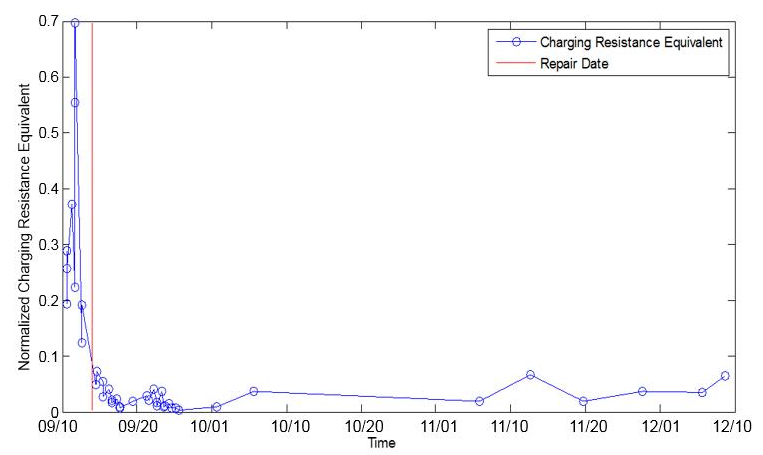

Figure 15 . The trend of the battery charging resistance equivalent from one field case (Case 8).

\section{Conclusion}

Battery and starter failures are common in the field and challenging to predict. Main reasons include, but are not limited to, part-to-part variations, non-linear system dynamics, limited onboard measurements, vulnerability to environment and usage dependent. In this work, the battery cranking resistance ratio and the battery charging resistance equivalent are identified as the fault signatures to predict the battery or starter failures. The robust algorithms to extract these fault signatures are developed and validated with large amount of the field data.

The proposed solution has been used in OnStar ${ }^{\mathrm{TM}}$ Proactive Alert service for selected MY16 or beyond GM North America vehicles and received very positive feedback from our customers. In the future, our research focus will be on the algorithm and architecture optimization to achieve better performance, and system efficiency.

\section{REFERENCES}

Bayir, R., \& Bay, O. (2004). Serial wound starter motor faults diagnosis using artificial neural network. IEEE International Conference on Mechatronics.

Berecibar, M., Gandiaga, I., Villarreal, I., Omar, N., Mierlo, J., \& Bossche, P. (2016). Critical review of state of health estimation methods of Li-ion batteries for real applications. Renewable and Sustainable Energy Reviews, vol. 56, pp. 572-587.

Coleman, M., Lee, C., Zhu, C., \& Hurley, W. G. (2007). State-of-Charge Determination From EMF Voltage Estimation: Using Impedance, Terminal Voltage, and Current for Lead-Acid and Lithium-Ion Batteries. IEEE Transactions on Industrial Electronics, vol. 54, no. 5, pp.2550-2557.

Cugnet, M., Sabatier, J., Laruelle, S., Grugeon, S., Sahut, B., Oustaloup, A., \& Tarascon, J.-M. (2010). On leadacid-battery resistance and cranking -capability estimation. IEEE Transactions on Industrial Electronics, vol. 57, no. 3, pp. 909-917.

Cuma, M., \& Koroglu, T. (2015). A comprehensive review on estimation strategies used in hybrid and battery electric vehicles. Renewable and Sustainable Energy Reviews, vol. 42, pp. 517-531.

Farmann, A., Waag, W., Marongui, A., \& Sauer, D. U. (2015). Critical review of on-board capacity estimation techniques for lithium-ion batteries in electric and hybrid electric vehicles. Journal of Power Sources, vol. 281, pp. 114-130.

Hu, X., Jiang, J., Cao, D., \& Egardt, B. (2016). Battery Health Prognosis for Electric Vehicles Using Sample Entropy and Sparse Bayesian Predictive Modeling. IEEE Transactions on Industrial Electronics, vol. 63, no. 4, pp. 2645-2656.

Pilatowicz, G., Marongiu, A., Drillkens, J., Sinhuber, P., \& Sauer, D. (2015). A critical overview of definitions and determination techniques of the internal 
resistance using lithium-ion, lead-acid, nickel metal-hydride batteries and electrochemical doublelayer capacitors as examples. Journal of Power Sources, vol. 296, pp. 365-376.

Schiffer, J., Sauer, D. U., Bindner, H., Cronin, T., Lundsager, P., \& Kaiser, R. (2007). Model prediction for ranking lead-acid batteries according to expected lifetiime in renewable energy systems and autonomous power-supply systems. Journal of Power Sources, vol. 168, pp. 66-78.

Shepherd, C. M. (1965). Design of primary and secondary cells. J. Electrochem. Soc., vol. 112, no. 7, pp. 657664.

Shin, K.-K., \& Sabahi-Kaviani, R. (2010). Diagnosis and Prognosis of Cranking Systems: A Parameter Based Approach. General Motors Global Research \& Development.

Shin, K.-K., \& Salman, M. A. (2010). Evidence theory based automotive battery health monitoring. $S A E$ International Journal of Passenger CarsElectronic and Electrical Systems, vol. 3, pp. 10-16.

Wang, Y., Fang, H., Wang, B., \& Liu, H. (2016). Highly precise determination of the state of charge of vehicular battery. Instrumentation Science \& Technology, vol. 44, no. 5, pp. 445-457.

Yan, W., Zhang, B., Wang, X., Dou, W., \& Wang, J. (2016). Lebesgue-Sampling-Based Diagnosis and Prognosis for Lithium-Ion Batteries. IEEE Transactions on Industrial Electronics, vol. 63 no. 3, pp. 1804-1812.

Zaidi, S., Aviyente, S., Salman, M., Shin, K.-K., \& Strangas, E. (2011). Prognosis of Gear Failures in DC Starter Motors Using Hidden Markov Models. IEEE Transactions on Industrial Electronics, vol. 58, no. 5, pp.1695-1706.

Zhang, F., Liu, G., Fang, L., \& Wang, H. (2012, 2). Estimation of Battery State of Charge With $\mathrm{H} \infty$ Observer: Applied to a Robot for Inspecting Power Transmission Lines. IEEE Transactions on Industrial Electronics, vol. 59 no. 2, pp. 1086-1095.

Zhang, X., Grube, R., Shin, K.-K., Salman, M., \& Conell, R. (2011). Parity-relation-based state-of-health monitoring of lead acid batteries for automotive applications. Control Engineering Practice, vol. 19 no. 6, pp. 555-563.

Zhang, Y., \& Du, X. (2014). Automatic field data analyzer for closed-loop vehicle design. Information Sciences, vol. 259, pp. 321-334.

Zhang, Y., Du, X., \& Salman, M. (2012). Peer-to-peer collaborative vehicle health management - the concept and an initial study. Annual Conference of Prognostics and Health Management Society 2012, 3. Minneapolis, Minnesota, USA.

Zhang, Y., Du, X., \& Salman, M. (2017). Battery state estimation with a self-evolving electrochemical ageing model. International Journal of Electrical Power \& Energy Systems, vol. 85, pp. 178-189.

\section{BIOGRAPHIES}

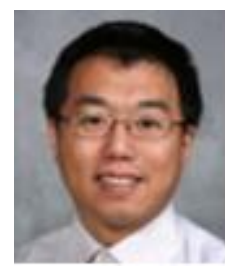

Xinyu Du received B.Sc. and M.Sc. degrees in automation from Tsinghua University, Beijing, China, in 2001 and 2004, respectively, and a Ph.D. in electrical engineering from Wayne State University, MI, USA, in 2012. He has been working at General Motors Global R\&D Center, Warren, MI, since 2010, and currently holds the senior researcher position in the vehicle system research lab. His research interests include fuzzy hybrid system, vehicle health management, deep learning and data analytics. He has published more than 30 peer review papers and holds 33 patents or patent applications. He has been serving as an associate editor for Journal of Intelligent and Fuzzy Systems from 2012 and IEEE Access from 2018. He received the Boss Kettering Award from General Motors for his contribution in integrated starting system prognosis in 2015.

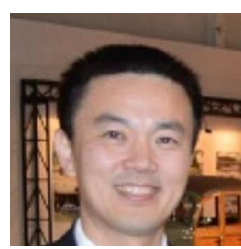

Yilu Zhang is a GM Technical Fellow and the group manager of vehicle health management at General Motors Global R\&D center, Warren, Michigan. He received B.S. and M.S. degrees in electrical engineering from Zhejiang University, China, in 1994, and 1997, respectively; and a Ph.D. in computer science from Michigan State University, East Lansing, MI, in 2002. His research interests include statistical pattern recognition, machine learning, signal processing, and their applications, including integrated vehicle health management and human machine interactions. Dr. Zhang's R\&D team worked with a cross-functional team to launch the industry-first OnStar ${ }^{\mathrm{TM}}$ Proactive Alerts service in 2015. This customer care service can predict when certain components need attention, and notify drivers before vehicle performance is affected. This technology is one of the supporting practices that won the INFORMS award for General Motors in 2016. 